\title{
The Quantitative Studies of some Chemical Constituents of the Mulberry Leaves. Part I.
}

\author{
The Study of the Cystine- and Cysteine- \\ Compounds in the Mulberry Leaves.
}

By

YUKitaRo KIshi.

(Received August 9 th, 1932).

\section{Résumé.}

(1) For the purpose of studing muiberry culture, I made a quantitative study of the cystine- and cysteine-compounds in the mulberry leaves, using Okuda's S. S. A. method ${ }^{(1)}$.

(2) Mulberry trees are so vigorous in growth that, even if the stems are cut off close at the stub twice or thrice in succession in a year, new buds form more readily and the leaves and stems thus produced grow more vigorously and luxuriantly than in the case of many other trees similarly treated; therefore, the quantity of the cystine- and cysteine-compounds in the fresh matter of the leaves is small in the buds and very young leaves, but increases in proportion to the growth of the leaves, and reaches the maximum quantity at the part where the action of the synthesis is most vigorous, and then decreases again in the fresh matter of the old leaves. In these compounds in the mulberry leaves, the $\mathrm{SH}$ form is found present in greater quantity than the S-S form at each stage of the growth of the leaves.

(3) The quantitative difference of the cystine- and cysteine-compounds contained in the leaves depends on the varieties of the mulberry trees; this is especially so in the young leaves. In the leaves of "Roso", the amount of these compounds tends to be smaller than in the leaves of other varieties; this is especially so in the young leaves used to feed young silkworms.

(4) My experiment has shown that a "hishage" stem (without leaves) of a mulberry tree contains a greater quantity of the cystine- and cysteinecompounds than a healthy stem (without leaves); while, on the contrary, the leaves of the "hishage" stem contains a smaller quantity of these compounds than the leaves of the healthy stem.

The "hishage" is an epithet given by many Japanese sericulturists to a

(1) Journal of the Department of Agriculture, Kyûshû Imperial University, Vol. 2, No. 5, (1929); also Journal of the Agricultural Chemical Society of Japan, 3, 1907, (1927). 
physiologically unsound stem. The "hishage" stem is flattened as if crushed, and is fatter in the affected parts than a healthy stem.

Addition:- In studying the amount of the cystine- and cysteine-compounds contained in the mulberry leaves, I have also examined the amount of these compounds contained in a silkworm made to abstain from food. I found that the quantity of the S-S form increases gradually, contrary to that of the $\mathrm{SH}$ form.

\title{
The Quantitative Studies of some Chemical Constituents of the Mulberry Leaves. Part II.
}

The Acid-base Balance of the Ash in the Mulberry Leaves.

\author{
By \\ YURITARO KISHI.
}

(Received August 9 th, 1932).

\section{Résumé.}

(1) I made a study of the acid-base balance of the ash in the mulberry leaves.

(2) The total alkalinity of the ash in the mulberry leaves was determined after incineration, with or without the addition of magnesium nitrate. In either instance, the alkalinity increased in proportion to the growth of the mulberry leaves, as the contents of the ash in the leaves increase in proportion to the growth.

(3) The total alkalinity of the ash in the mulberry leaves treated with magnesium nitrate is generally a little less than that obtained by the ordinary method.

(4) The alkalinity of the ash which is soluble in water decreased in proportion to the growth of the mulberry leaves, as against the total alkalinity in the two instances mentioned above, which increased in proportion to the growth. Consequently, the alkaline ash constituents which is insoluble in water showed an increase in proportion to the growth of the leaves. 